\title{
AVALIAÇÃO DAS CONDIÇÕES HIGIÊNICO- SANITÁRIAS EM UNIDADES DE ALIMENTAÇÃO ESCOLAR DA REDE MUNICIPAL DE UM MUNICÍPIO DO VALE DO RIO PARDO, RIO GRANDE DO SUL
}

\author{
Bruna dos Santos Rasquinha1 ${ }^{1}$, Gabriela Quadros Nunes², Fernanda Scherer Adami², \\ Patricia Fassina ${ }^{4}$
}

\begin{abstract}
Resumo: Objetivos: Avaliar as condições higiênico-sanitárias de Unidades de Alimentação e Nutrição (UANs) de escolas de educação infantil e ensino fundamental, bem como comparar as condições sanitárias entre as escolas. Metodologia: Estudo observacional transversal, desenvolvido entre agosto e setembro de 2015 em 24 UANs escolares de um município do Vale do Rio Pardo, Rio Grande do Sul, por meio da Portaria no 78/2009, a qual constitui um checklist de 153 critérios distribuídos em 12 categorias. Os dados foram analisados no software Statistical Package for the Social Sciences da IBM ${ }^{\circledR}$, versão 20.0, considerando o nível de significância de $5 \%(\mathrm{p}<0,05)$. Resultados: Maiores percentuais de conformidade higiênico-sanitários foram encontrados nas categorias Responsabilidade $(100,00 \pm$ 0,00\%), Manejo de Resíduos (90,28 \pm 2,80\%), Matérias Primas, Ingredientes e Embalagens (86,46 \pm 4,12\%), Abastecimento de Água (82,81 $\pm 19,09 \%)$, Higienização de Instalações, Equipamentos, Móveis e Utensílios $(80,73 \pm 8,22 \%)$, Preparação do Alimento $(79,35 \pm 2,94 \%)$ e Exposição ao Consumo do Alimento preparado $(76,35 \pm 3,83 \%)$. Identificou-se melhor adequação sanitária nas escolas de educação infantil para as categorias Edificação das Instalações, Equipamentos, Móveis e Utensílios (72,20 \pm 0,91\% e $67,70 \pm 4,47 \%, p=0,002)$ e Manipuladores $(73,86 \pm 1,93 \%$ e 71,65 $\pm 2,98 \%, p=0,043)$. As escolas de ensino fundamental apresentaram melhores condições sanitárias para a categoria Exposição ao Consumo do Alimento Preparado (75,00 $\pm 0,00 \%$ e 77,71 $\pm 5,16 \%, p=0,005)$. Conclusões: As UANs escolares apresentaram condições higiênico-sanitárias para alguns critérios da legislação, enquanto que outros não estavam em conformidade. As escolas de educação infantil apresentaram melhores condições sanitárias nas categorias Edificação, Instalações, Equipamentos, Móveis e Utensílios e Manipuladores, enquanto que as escolas de ensino fundamental maior conformidade para a categoria Exposição ao Consumo do Alimento Preparado.
\end{abstract}

Palavras-chave: Boas Práticas de Manipulação. Serviços de alimentação. Refeições. Alimentos.

1 Acadêmica do curso de Nutrição, Univates.

2 Acadêmica do curso de Nutrição, Univates.

3 Doutora em Ciências da Saúde, docente do curso de Nutrição, Univates.

4 Mestre em Ambiente e Desenvolvimento, docente do curso de Nutrição, Univates. 


\title{
EVALUATION OF HYGIENIC-SANITARY CONDITIONS IN SCHOOL FEEDING UNITS FROM A MUNICIPAL NETWORK IN A CITY OF VALE DO RIO PARDO, RIO GRANDE DO SUL
}

\begin{abstract}
Objective: To evaluate the hygienic-sanitary conditions of Food and Nutrition Units (FNUs) of kindergarten and elementary schools, as well as to compare sanitary conditions among schools. Methodology: Cross-sectional observational study, developed in the period of August to September 2015 in 24 school feeding and nutrition units of a municipality in the Rio Pardo Valley, Rio Grande do Sul, through Portaria no 78/2009, which is a checklist of 153 criteria distributed in 12 categories. Data were analyzed in the IBM ${ }^{\circledR}$ Statistical Package for the Social Sciences, version 20.0, considering the level of significance of 5\% ( $p<0.05)$. Results: The highest percentages of hygienic-sanitary compliance were found in the categories Responsibility $(100.00 \pm 0.00 \%)$, Handling of Residues $(90.28 \pm 2.80 \%)$, Raw Materials, Ingredients and Packaging $(86.46 \pm 4,12 \%)$, Water Supply $(82.81 \pm 19.09 \%)$, Sanitation Facilities, Equipment, Furniture and Utensils (80.73 $\pm 8.22 \%)$, Food Preparation (79.35 $\pm 2.94 \%)$ and Food Consumption Exposure (76.35 \pm 3.83\%). It was identified a better sanitary adequacy in kindergarten schools for the categories of Facilities, Equipment, Furniture and Utensils $(72,20 \pm 0,91 \%$ and $67,70 \pm 4,47 \%, p=0,002)$ and Manipulators $(73,86 \pm 1.93 \%$ and $71.65 \pm 2.98 \%, \mathrm{p}=0.043$ ). The elementary schools had better sanitary conditions for the category of Exposure to Consumption of Prepared Food $(75.00 \pm 0.00 \%$ and $77.71 \pm 5.16 \%, p=$ 0.005). Conclusion: School FNUs presented hygienic-sanitary conditions for some of the criteria in the legislation, while others were not in compliance. The elementary schools had better sanitary conditions in the categories Building, Facilities, Equipment, Furniture and Utensils and Handlers, while elementary schools had greater conformity for the category Exposure to Consumption of Prepared Food.
\end{abstract}

Keywords: Good Handling Practices. Food Services. Meals. Food.

\section{INTRODUÇÃO}

O Programa Nacional de Alimentação Escolar (PNAE) objetiva o fornecimento de uma alimentação saudável, diversificada, balanceada, segura, de qualidade e que atenda todas as necessidades nutricionais de crianças, adolescentes e adultos matriculados na educação básica. Com essa oferta, o programa visa a contribuir para o desenvolvimento, crescimento, aprendizagem e melhora do rendimento escolar do aluno, além de incentivar a prática alimentar saudável (BRASIL, 2015a).

O PNAE exige que todos os alimentos adquiridos para a alimentação escolar estejam dentro dos padrões estabelecidos pela Agência Nacional de Vigilância Sanitária (ANVISA) e que as escolas adotem medidas adequadas e seguras de aquisição, transporte, armazenamento, manipulação e distribuição para garantir a qualidade da refeição oferecida (BRASIL, 2013).

Neste sentido, é imprescindível o cumprimento das legislações sanitárias para as Unidades de Alimentação e Nutrição (UANs), principalmente nos serviços que oferecem refeições a crianças e adolescentes, pois os procedimentos relacionados às boas práticas de manipulação estão diretamente relacionados à proteção contra as doenças 
transmitidas por alimentos (DTAs) (SILVA et al., 2016). Estas podem ser contraídas por qualquer pessoa através do consumo de alimentos e/ou água contaminados por bactérias, vírus e/ou parasitas patogênicos (PORTAL DA SAÚDE, 2015).

As DTAs podem ser caracterizadas como infecções de origem alimentar quando são causadas pela ingestão de micro-organismo patogênicos invasivos capazes de penetrar e invadir os tecidos de um indivíduo; como toxinfecções alimentares quando são causadas por micro-organismo toxigênicos que liberam suas toxinas dentro do organismo da pessoa; ou como intoxicações alimentares quando são causadas pela ingestão de toxinas pré-formadas por micro-organismo patogênicos nos alimentos. Dentre os sintomas mais comuns estão dor abdominal, vômito, diarreia, perda de peso e presença ou não de febre (BRASIL, 2014). Entre os anos de 2000 e 2015, os locais de maior ocorrência de surtos de DTAs no Brasil foram, respectivamente, residências $(38,4 \%)$, restaurantes/padarias (15,5\%) e creches/escolas (8,7\%) (BRASIL, 2015b).

Para um alimento ser considerado seguro, ele deve estar livre de contaminantes químicos, físicos e biológicos (SILVA; CARDOSO, 2011). Para evitar a contaminação dos alimentos e garantir a segurança dos mesmos, devem ser adotadas medidas preventivas desde a aquisição das matérias-primas, para as quais devem ser especificados critérios de avaliação, bem como para a seleção de fornecedores pelos serviços de alimentação (BRASIL, 2004a). Além disso, devem ser mantidas medidas para a higienização completa e eficaz das instalações, equipamentos, móveis e utensílios (BRASIL, 2004b).

Os maiores fatores associados ao risco de contaminação dos alimentos nas escolas são o tempo entre o preparo e a distribuição e as condições de higiene dos locais. Neste sentido, as medidas preventivas abrangem três aspectos principais dentro das cozinhas e refeitórios das UANS, sendo o ambiente, os alimentos e os manipuladores de alimentos (OLIVEIRA; BRASIL; TADDEI, 2008).

Assegurar a qualidade do alimento oferecido aos alunos, durante o período escolar, é uma maneira de investir no desenvolvimento e na promoção da saúde das crianças, principalmente pelo fato de que, para algumas famílias, a refeição oferecida através da alimentação escolar é o único alimento que a criança recebe no dia (SILVA; CARDOSO, 2011). Portanto, o profissional nutricionista tem como função contribuir para a saúde de indivíduos e coletividades, devendo buscar continuamente o aperfeiçoamento técnico-científico (BRASIL, 2004c).

Dentre as suas responsabilidades profissionais na área de alimentação coletiva, o nutricionista deve planejar, organizar, dirigir, supervisionar e avaliar os serviços de alimentação e nutrição. Entre suas atribuições, cabe a ele estabelecer e implantar procedimentos operacionais padronizados e métodos de controle e qualidade de alimentos, em conformidade com a legislação vigente, além de planejar, orientar e supervisionar as atividades de seleção, compra, armazenamento, produção e distribuição dos alimentos, zelando pela qualidade dos produtos, observando as boas práticas higiênicas e sanitárias (BRASIL, 2005). 
Diante do exposto, este estudo teve como objetivo avaliar as condições higiênicosanitárias de UANs escolares, bem como comparar as condições sanitárias entre as escolas de educação infantil e ensino fundamental.

\section{METODOLOGIA}

Estudo de caráter observacional transversal, desenvolvido no período de agosto a setembro de 2015 em 24 UANs, sendo 12 escolas de educação infantil e 12 escolas de ensino fundamental, localizadas em um município do Vale do Rio Pardo, interior do Rio Grande do Sul.

Para a avaliação das condições higiênico-sanitárias das UANs foi utilizada a Portaria $\mathrm{n}^{\circ}$ 78, de 30 de janeiro de 2009 (RIO GRANDE DO SUL, 2009), a qual se constitui de um checklist que aprova a lista de verificação em boas práticas para serviços de alimentação dotada de um total de 153 critérios distribuídos em 12 categorias distintas: 1. Edificação, instalações, equipamentos, móveis e utensílios ( $\mathrm{n}=34$ critérios); 2. Higienização das instalações, equipamentos, móveis e utensílios ( $\mathrm{n}=17$ critérios); 3. Controle integrado de vetores e pragas urbanas ( $n=7$ critérios); 4 . Abastecimento de água ( $\mathrm{n}=9$ critérios); 5 . Manejo de resíduos ( $\mathrm{n}=3$ critérios); 6. Manipuladores $(\mathrm{n}=15$ critérios); 7. Matérias-primas, ingredientes e embalagens ( $\mathrm{n}=12$ critérios); 8. Preparação do alimento ( $\mathrm{n}=26$ critérios); 9. Armazenamento e transporte do alimento preparado ( $\mathrm{n}=6$ critérios); 10. Exposição ao consumo do alimento preparado ( $\mathrm{n}=9$ critérios); 11. Documentação e registo ( $\mathrm{n}=8$ critérios); e 12. Responsabilidade ( $\mathrm{n}=7$ critérios).

Cada critério avaliado foi assinalado com um " $\mathrm{x}$ " nas colunas "Sim" ou "Não" do referido checklist. Quando "Sim", o critério atendeu a conformidade do local em boas práticas e, quando "Não", o critério não atendeu a legislação. Além das colunas "Sim" e "Não", havia ainda a opção de marcar um "x" na coluna "NA", em caso do critério não condizer com a realidade da UAN avaliada. Por este motivo, a categoria 9 "armazenamento e transporte do alimento preparado" foi excluída do estudo por não se aplicar à realidade das UANs estudadas.

O presente estudo foi aprovado pelo Comitê de Ética em Pesquisa (COEP) da Univates conforme a Resolução 466/12. As visitas realizadas nas UANs não foram agendadas para que não houvesse modificação na rotina de trabalho dos manipuladores de alimentos, visto que as práticas destes foram analisadas em uma das categorias do checklist.

Os manipuladores de alimentos assinaram o Termo de Consentimento Livre e Esclarecido (TCLE) em duas vias, antes e após a verificação das boas práticas, a fim de evitar mudanças de comportamento por parte dos mesmos, ficando uma via para o voluntário e outra para o pesquisador.

O TCLE anterior à aplicação do checklist abordou os procedimentos e objetivos do estudo de forma geral e o TCLE posterior à verificação das boas práticas informou 
sobre a observação dos manipuladores quanto à presença de doenças de pele das mãos e unhas, lesões e/ou sintomas que poderiam comprometer a qualidade higiênicosanitária dos alimentos; uniformização; apresentação pessoal e práticas de manipulação de alimentos, cabendo a eles manter ou retirar o seu consentimento. Todos os manipuladores mantiveram o seu consentimento após a segunda aplicação o TCLE.

Os dados foram analisados no software Statistical Package for the Social Sciences (SPSS) da IBM ${ }^{\circledR}$, versão 20.0 e adotou-se o nível de significância de $5 \%(\mathrm{p}<0,05)$. Foram realizadas estatísticas univariadas descritivas (médias, variâncias, desvios-padrão e frequências) e bivariadas (testes t de Student, Mann Whitney, correlações de Pearson e Spearman). Utilizou-se o teste de Shapiro-Wilk para avaliar se as variáveis seguiam a distribuição normal. Para as que seguiam distribuição normal, os resultados foram analisados por testes paramétricos, já as que não seguiam por testes não-paramétricos. Os testes t de Student foram aplicados para amostras independentes, Mann Whitney para comparar os percentuais de conformidade encontrados para cada item da Portaria 78/2009 entre as escolas de ensino infantil e ensino fundamental e os testes de correlação de Pearson e Spearman foram aplicados para avaliar a associação entre os itens.

\section{RESULTADOS}

Das 24 escolas avaliadas, os maiores percentuais de conformidade de condições higiênico-sanitárias foram encontrados nas categorias Responsabilidade (100,00 \pm 0,00\%), Manejo de Resíduos (90,28 $\pm 2,80 \%$ ), Matérias Primas, Ingredientes e Embalagens (86,46 $\pm 4,12 \%)$, Abastecimento de Água (82,81 $\pm 19,09 \%)$, Higienização de Instalações, Equipamentos, Móveis e Utensílios (80,73 \pm 8,22\%), Preparação do Alimento (79,35 $\pm 2,94 \%)$ e Exposição ao Consumo do Alimento preparado (76,35 \pm $3,83 \%)$. Os demais itens tiveram conformidade abaixo de $75 \%$ (Tabela 1 ).

Tabela 1. Percentual de conformidade das escolas segundo os critérios da Portaria 78/2009

\begin{tabular}{l|c|c}
\hline Itens $[\boldsymbol{n}$ 24] & Variância & \% (Média \pm DP) \\
\hline Edificação, Instalações, Equipamentos, Móveis e Utensílios & $62-75$ & $69,95 \pm 3,90$ \\
\hline Higienização de Instalaç̃̃es, Equipamentos, Móveis e Utensilios & $63-94$ & $80,73 \pm 8,22$ \\
\hline Controle de Pragas & $57-57$ & $57,10 \pm 0,00$ \\
\hline Abastecimento de Água & $63-100$ & $82,81 \pm 19,09$ \\
\hline Manejo de Resíduos & $33-100$ & $90,28 \pm 20,80$ \\
\hline Manipuladores & $67-80$ & $72,75 \pm 2,71$ \\
\hline Matérias Primas, Ingredientes e Embalagens & $83-92$ & $86,46 \pm 4,12$ \\
\hline Preparação do Alimento & $78-87$ & $79,35 \pm 2,94$ \\
\hline
\end{tabular}




\begin{tabular}{l|c|c}
\hline Itens $[\boldsymbol{n}$ 24] & Variância & \% (Média \pm DP) \\
\hline Exposição ao Consumo do Alimento preparado & $63-80$ & $76,35 \pm 3,83$ \\
\hline Documentação e Registro & $43-43$ & $42,85 \pm 0,00$ \\
\hline Responsabilidade & $100-100$ & $100,00 \pm 0,00$ \\
\hline
\end{tabular}

Resultados descritos em variância, percentuais (\%), média e desvio-padrão (DP).

Fonte: dos autores.

Comparando as escolas de educação infantil e ensino fundamental, o percentual de adequação à Portaria 78/2009 (RIO GRANDE DO SUL, 2009) foi significativamente maior para as categorias Edificação, Instalações, Equipamentos, Móveis e Utensílios $(72,20 \pm 0,91 \%$ e $67,70 \pm 4,47 \%, p=0,002)$ e Manipuladores $(73,86 \pm 1,93 \%$ e 71,65 $\pm 2,98 \%, p=0,043)$ nas escolas de ensino infantil e fundamental, respectivamente. Já, para o item Exposição ao Consumo do Alimento preparado, a conformidade foi significativamente maior entre as escolas de educação infantil em relação ao ensino fundamental $(75,00 \pm 0,00 \%$ e $77,71 \pm 5,16 \%, p=0,005)$, respectivamente (Tabela 2).

Tabela 2. Comparação da conformidade entre as escolas de educação infantil e ensino fundamental

\begin{tabular}{l|c|c|c}
\hline Itens [n 24] & $\begin{array}{c}\text { Ensino Infantil } \\
\text { \% Conformidade } \\
\text { Média } \pm \text { DP }\end{array}$ & $\begin{array}{c}\text { Ensino Fundamental } \\
\text { \% Conformidade } \\
\text { Média } \pm \text { DP }\end{array}$ & p \\
\hline $\begin{array}{l}\text { Edificação, Instalações, } \\
\text { Equipamentos, Móveis e Utensílios }\end{array}$ & $72,20 \pm 0,91^{\mathrm{A}}$ & $67,70 \pm 4,47^{\mathrm{A}}$ & 0,002 \\
\hline $\begin{array}{l}\text { Higienização de Instalações, } \\
\text { Equipamentos, Móveis e Utensílios }\end{array}$ & $81,21 \pm 4,62^{\mathrm{B}}$ & $80,21 \pm 10,93^{\mathrm{B}}$ & 0,764 \\
\hline Controle de Pragas & $57,10 \pm 0,00^{\mathrm{B}}$ & $57,10 \pm 0,00^{\mathrm{B}}$ & 1,00 \\
\hline Abastecimento de Água & $87,50 \pm 18,46^{\mathrm{B}}$ & $78,13 \pm 19,31^{\mathrm{B}}$ & 0,237 \\
\hline Manejo de Resíduos & $97,22 \pm 9,62^{\mathrm{B}}$ & $83,34 \pm 26,59^{\mathrm{B}}$ & 0,103 \\
\hline Manipuladores & $73,86 \pm 1,93^{\mathrm{A}}$ & $71,65 \pm 2,98^{\mathrm{A}}$ & 0,043 \\
\hline $\begin{array}{l}\text { Matérias Primas, Ingredientes e } \\
\text { Embalagens }\end{array}$ & $86,11 \pm 4,11^{\mathrm{B}}$ & $86,81 \pm 4,29^{\mathrm{B}}$ & 0,689 \\
\hline Preparação do Alimento & $79,71 \pm 3,39^{\mathrm{B}}$ & $78,99 \pm 2,51^{\mathrm{B}}$ & 0,557 \\
\hline $\begin{array}{l}\text { Exposição ao Consumo do } \\
\text { Alimento preparado }\end{array}$ & $75,00 \pm 0,00^{\mathrm{A}}$ & $77,71 \pm 5,16^{\mathrm{A}}$ & 0,005 \\
\hline Documentação e Registro & $42,85 \pm 0,00^{\mathrm{B}}$ & $42,85 \pm 0,00^{\mathrm{B}}$ & 1,00 \\
\hline Responsabilidade & $100,00 \pm 0,00^{\mathrm{B}}$ & $100,00 \pm 0,00^{\mathrm{B}}$ & 1,00 \\
\hline
\end{tabular}

Testes $\mathrm{t}$ de Student e Mann-Whitney para comparação entre as médias de conformidade segundo as escolas, considerando significativo $\mathrm{p}<0,05$ (5\%). Resultados descritos em percentuais (\%), média e desvio-padrão (DP). A = resultados significativos. $\mathrm{B}=$ resultados não significativos.

Fonte: dos autores. 


\section{DISCUSSÃO}

$\mathrm{Na}$ categoria Edificação, Instalações, Equipamentos, Móveis e Utensílios do presente estudo, não foi obtido maior percentual de conformidade devido a inexistência de separações por meios físicos que evitam a contaminação cruzada entre as atividades de pré-preparo, preparo e higienização, sendo estes realizados no mesmo local e ao mesmo tempo. Esta prática facilita a contaminação pelo contato entre alimentos crus e cozidos e até mesmo com os produtos saneantes utilizados na higienização. Outros fatores envolvidos com a não conformidade relacionam-se a inexistência de portas com fechamento automático e luminárias com proteção contra explosões e quedas acidentais na área de preparação e armazenamento, além de lavatórios exclusivos para higienização das mãos na área de manipulação de alimentos, sendo melhores adequações observadas nas escolas de educação infantil.

Problemas estruturais similares e em percentuais superiores ao atual estudo foram encontrados por Cardoso et al. (2010) em UANs escolares, as quais também não apresentavam áreas distintas para o preparo dos alimentos, luminárias com proteção e ausência de lavatório exclusivo para higienização das mãos. Lopes et al. (2015) também encontraram luminárias sem proteção em UANs escolares e classificaram a categoria de estrutura das instalações como situação de risco.

A proteção das luminárias é importante para evitar quedas ou explosões acidentais podendo resultar em risco físico aos alimentos, contaminando-os com resíduos de vidro e metal, além de aumentar os riscos de acidente de trabalho (LOPES et al., 2015). A existência de lavatórios exclusivos para higienização das mãos na área de manipulação de alimentos é necessária para que os manipuladores possam lavar cuidadosamente as mãos ao chegar ao trabalho, antes e após manipular alimentos, após qualquer interrupção do serviço, toque a materiais contaminados, uso de sanitários e sempre que se fizer necessário, a fim de evitar a contaminação (GOMES; CAMPOS; MONEGO, 2012).

Para a categoria Controle Integrado de Pragas, tanto nas escolas de educação infantil quanto de ensino fundamental não atingiu um bom percentual de conformidade por não apresentarem registros que comprovam a aplicação de controle químico de vetores e pragas urbanas por empresas especializadas. Esse fato indicou que o controle químico de vetores e pragas não foi realizado no tempo previsto pela legislação, contudo, não foi observado a presença de insetos e de outras pragas nas UANs avaliadas.

Lopes et al. (2015) identificaram resultado diferente ao encontrado no presente estudo, ao observarem que, em algumas unidades escolares, não somente o controle de insetos e pragas urbanas não era realizado com frequência, mas também a ausência de medidas preventivas importantes, como ausência de telas nas janelas e teto descoberto, além da presença de insetos e teias de aranha. Cardoso et al. (2010) também observaram presença de insetos e vestígios deles em quase metade das UANs escolares avaliadas, devido a presença de lixo e de material em desuso nos arredores, bem como ausência de barreiras de proteção contra a entrada desses animais. Segundo a legislação, a 
edificação, as instalações, os equipamentos, os móveis e os utensílios devem estar livres de vetores e pragas urbanas. Por este motivo, as unidades devem adotar um conjunto de ações eficazes e contínuas de controle desses animais, com o objetivo de impedir a atração, o abrigo, o acesso e a proliferação dos mesmos (BRASIL, 2004b).

Em relação aos manipuladores, não foram observadas melhores adequações nas UANs avaliadas devido a presença de irregularidades, como a inexistência de registro de exames periódicos de saúde dos manipuladores, indicando a possível falta de controle da saúde dos mesmos. Identificou-se também a ausência de cartazes de orientação aos manipuladores sobre a correta higienização das mãos, sendo melhores adequações observadas nas escolas de ensino infantil. Semelhante ao resultado encontrado no presente estudo, Gomes, Campos e Monego (2012), identificaram que não havia realização de exames periódicos pelos manipuladores. No estudo de Cardoso et al. (2010), foi observada a inexistência de capacitação semestral dos manipuladores. Segundo as legislações específicas para as boas práticas em serviço de alimentação, o controle da saúde do manipulador deve ser realizado de acordo com a legislação específica e os mesmos devem ser capacitados periodicamente (BRASIL, 2004c; CARDOSO et al., 2010). As capacitações constantes visam conscientizar os manipuladores de alimentos sobre a sua responsabilidade e aos cuidados que devem ter no preparo de alimentos, de forma a qualificá-los para a segurança e qualidade das refeições produzidas (SILVA et al., 2016). Assim, o conhecimento acerca das boas práticas de manipulação de alimentos se torna fundamental para contribuir para a melhoria da execução do PNAE e garantir a produção de refeições adequadas e saudáveis, protegendo a saúde dos alunos (GOMES; CAMPOS; MONEGO, 2012).

No atual estudo, na categoria Exposição ao consumo do alimento preparado observou-se que todas as escolas possuíam refeitório organizado e em adequadas condições higiênico-sanitárias e que apenas 62,5\% ( $\mathrm{n}=15)$ delas apresentaram equipamento de exposição do alimento preparado, sendo 12 (80\%) destas, as escolas de educação infantil. A conformidade da categoria foi significativamente maior entre as escolas de ensino fundamental, devido às escolas de ensino infantil não apresentarem registro de temperatura do equipamento de exposição verificados, datados e rubricados. As escolas de ensino fundamental que não possuíam equipamento de exposição do alimento preparado serviam uma refeição por dia aos alunos, como lanche da manhã ou tarde, e justificaram não haver necessidade e condições financeiras para a aquisição do mesmo. Em contrapartida, no estudo de Cardoso et al., (2010), foi observado que a maioria das escolas não possuíam refeitórios, sendo os alimentos consumidos no pátio ou na sala de aula. Os equipamentos necessários à exposição ou distribuição de alimentos preparados, sob temperaturas controladas, devem estar em adequado estado de higiene, conservação e funcionamento. A temperatura desses equipamentos deve ser regularmente monitorada de forma a garantir que o alimento fique na faixa de temperatura segura durante o período de distribuição (BRASIL, 2004b).

Em relação à categoria Documentação e Registro identificou-se um baixo percentual de conformidade devido às escolas não possuírem o Manual de Boas Práticas 
(MBP) e os Procedimentos Operacionais Padronizados (POP). O estudo de Cardoso et al. (2010), que avaliou escolas municipais e estaduais, também identificou que nenhuma delas possuía MBP e que o acompanhamento por nutricionistas era insuficiente. Oposto ao resultado encontrado no presente estudo, Silva et al. (2016), identificaram, em seu estudo, uma maior adequação, visto que todas as escolas possuíam MBP e POP, porém esses documentos não estavam disponíveis aos colaboradores e órgãos fiscalizadores, e ainda muitos diretores e manipuladores de alimentos desconheciam seu conteúdo. Segundo a legislação, os serviços de alimentação devem dispor de MBP e de POP, sendo que os mesmos devem estar acessíveis aos funcionários da UAN e disponíveis à autoridade sanitária, quando requerido. Os POP devem ser aprovados, datados e assinados pelo responsável do estabelecimento e devem conter as instruções sequenciais das operações e a frequência de execução, especificando o nome, o cargo e/ou a função dos responsáveis pelas atividades (BRASIL, 2004b).

Durante as visitas nas UANs não foi possível observar todas as etapas realizadas durante a produção dos alimentos, desde a aquisição da matéria-prima até a distribuição de refeições. Quando a impossibilidade da observação de alguns critérios contidos no checklist, a conformidade ou não conformidade, foi registrada segundo o relato dos manipuladores dos locais, podendo este fato ser considerado uma limitação do presente estudo.

\section{CONCLUSÕES}

As UANs escolares apresentaram melhores condições higiênico-sanitárias para as categorias Responsabilidade; Manejo de Resíduos; Matérias Primas, Ingredientes e Embalagens; Abastecimento de Água; Higienização de Instalações, Equipamentos, Móveis e Utensílios; Preparação do Alimento e Exposição ao Consumo do Alimento preparado; enquanto que as categorias Edificação, Instalações, Equipamentos, Móveis e Utensílios; Controle de Pragas; Manipuladores; Documentação e Registro apresentaram menores índices de adequação por apresentarem critérios em não conformidade com a legislação.

Comparando as condições sanitárias entre as escolas de educação infantil e ensino fundamental, as categorias Edificação, Instalações, Equipamentos, Móveis e Utensílios e Manipuladores apresentaram melhores condições sanitárias para as escolas de educação infantil, porém houve maior conformidade nas escolas de ensino fundamental para a categoria Exposição ao Consumo do Alimento Preparado.

É importante ressaltar que a execução das boas práticas em serviços de alimentação tem o papel de garantir a qualidade dos alimentos oferecidos e que a implementação do MBP é essencial para obter conformidade com a legislação sanitária neste tipo de serviço. Acredita-se que muitas inconformidades podem ser sanadas com a atuação em número suficiente de profissional nutricionista, visto que seu papel é de suma importância no segmento de alimentação coletiva. 


\section{REFERÊNCIAS}

BRASIL. Agência Nacional de Vigilância Sanitária. Cartilha sobre Boas Práticas para serviços de alimentação: Resolução-RDC no 216/2004. Brasília, 2004a. Disponível em: <http://www.anvisa.gov.br/divulga/public/alimentos/cartilha_gicra_final.pdf >. Acesso em: 13 dez. 2017.

BRASIL. Ministério da Educação. Cartilha Nacional da Alimentação Escolar. Brasília, 2015a. Disponível em: <https://www.google.com.br/search?biw=1354\&bih=586\&ei=tncxWoq WIcmywATZ2rOICA\&q=Cartilha+Nacional+da+Alimenta\%C3\%A7\%C3\%A3o+Escolar\& oq=Cartilha+Nacional + da + Alimenta\%C3\%A7\%C3\%A3o+Escolar\&gs_1=psy-ab.3..014.11063 .11999.0.12728.3.3.0.0.0.0.119.223.0j2.3.0....0...1c.1.64.psy-ab..0.2.222.0..0i131k1j0i67k1.96. caaXJQt2jlw>. Acesso em: 13 dez. 2017.

BRASIL. Ministério da Saúde. Vigilância Epidemiológica das Doenças Transmitidas por Alimentos- VE-DTA. São Paulo, 2014. Disponível em: <http://www.anrbrasil.org.br/new/ pdfs/2014/3_PAINEL_1_ApresentacaoRejaneAlvesVigilanciaEpidemiologica-VE-DTAAgosto_2014_PDF.pdf> Acesso em: 13 dez. 2017.

BRASIL. Ministério da Saúde. Vigilância Epidemiológica das Doenças Transmitidas por Alimentos-VE-DTA. São Paulo, 2015b. Disponível em: <http://u.saude.gov.br/images/ pdf/2015/novembro/09/Apresenta----o-dados-gerais-DTA-2015.pdf> Acesso em: 13 dez. 2017.

BRASIL. Resolução CFN no 334/2004. Conselho Federal de Nutricionistas. Brasília, DF, 10 mai. 2004c. Disponível em: <http://www.cfn.org.br/novosite/pdf/res/2000_2004/res334. pdf> Acesso em: 13 dez. 2017.

BRASIL. Resolução CFN no 380/2005. Conselho Federal de Nutricionistas. Brasília, DF, 09 dez. 2005. Disponível em: <http://www.cfn.org.br/novosite/pdf/res/2005/res380.pdf> Acesso em: 13 dez. 2017.

BRASIL. Resolução no 26, de 17 de junho de 2013. Programa Nacional de Alimentação Escolar. Diário Oficial da União. Brasília, DF,17 jun. 2013. Disponível em: <https://www. google.com.br/search?biw=1354\&bih=586\&ei=f3gxWtToloiRwgTu1bBo\&q=Resolu\%C3\%A7 $\% \mathrm{C} 3 \% \mathrm{~A} 3 \mathrm{o}+\mathrm{n} \% \mathrm{C} 2 \% \mathrm{BA}+26 \% 2 \mathrm{C}+\mathrm{de}+17+\mathrm{de}+\mathrm{junho}+\mathrm{de}+2013 \&$ oq $=$ Resolu $\% \mathrm{C} 3 \% \mathrm{~A} 7 \% \mathrm{C} 3 \% \mathrm{~A} 3 \mathrm{o}$ $+\mathrm{n} \% \mathrm{C} 2 \% \mathrm{BA}+26 \% 2 \mathrm{C}+\mathrm{de}+17+\mathrm{de}+\mathrm{junho}+\mathrm{de}+2013 \& \mathrm{gs} \_1=$ psy-ab.3...35553.35553.0.36007.1.1.0. 0.0.0.108.108.0j1.1.0...0...1c.1.64.psy-ab..0.0.0...0._zpi28vxmX4> Acesso em: 13 dez. 2017.

BRASIL. Resolução no 216, de 15 de setembro de 2004. Diário Oficial da União. Brasília, DF, 16 set. 2004b. Disponível em: <http://portal.anvisa.gov.br/documents/33916/388704/ RESOLU\%25C3\%2587\%25C3\%2583O-RDC\%2BN\%2B216\%2BDE\%2B15\%2BDE\%2BSE TEMBRO\%2BDE\%2B2004.pdf/23701496-925d-4d4d-99aa-9d479b316c4b> Acesso em: 13 dez. 2017.

CARDOSO, R. C. V. et al. Programa nacional de alimentação escolar: há segurança na produção de alimentos em escolas de Salvador (Bahia)? Revista de Nutrição, v. 23, n. 5, p. 801-812, 2010. 
GOMES, N. A. A. A.; CAMPOS, M. R. H.; MONEGO, E. T. Aspectos higiênicosanitários no processo produtivo dos alimentos em escolas públicas do Estado de Goiás, Brasil. Revista de Nutrição, v. 25, n. 4, p. 473-485, 2012.

LOPES, A. C. C. et al. Avaliação das Boas Práticas em unidades de alimentação e nutrição de escolas públicas do município de Bayeux, PB, Brasil. Ciência Saúde Coletiva, v. 20, n. 7, p. 2267-2275, 2015.

OLIVEIRA, M. N.; BRASIL, A. L. D.; TADDEI, J. A. A. C. Avaliação das condições higiênico-sanitárias das cozinhas de creches públicas e filantrópicas. Ciência Saúde Coletiva, v. 13, n. 3, p. 1051-1060, 2008.

PORTAL DA SAÚDE. Secretaria de Vigilância em Saúde: Doenças Transmitidas por alimentos (DTA). Brasília, DF, 2015. Disponível em: <http://portalsaude.saude.gov.br/index. $\mathrm{php} / \mathrm{o}-\mathrm{ministerio/principal/secretarias/svs/doencas-transmitidas-por-alimentos-dta}>$ Acesso em: 13 dez 2017.

RIO GRANDE DO SUL. Portaria no 78, de 30 de janeiro de 2009. Secretaria da Saúde. Porto Alegre, RS, 28 jan. 2009. Disponível em: <http://www.saude.rs.gov.br/ upload/1365096500_portaria\%2078_09.pdf> Acesso em: 13 dez. 2016.

SILVA, L. M. et al. Avaliação das condições higiênicas de escolas de ensino infantil e fundamental por meio da aplicação de listas de verificação. Segurança Alimentar e Nutricional, v. 23, n. 1, p. 837-848, 2016.

SILVA, V. R.; CARDOSO, R. C. V. Controle da qualidade higiênico-sanitária na recepção e no armazenamento de alimentos: um estudo em escolas públicas municipais de Salvador, Bahia. Segurança Alimentar e Nutricional, v. 18, n. 1, p. 46-57, 2011. 